\title{
INFLUENCE OF A SPIRAL GRAVITATIONAL FIELD ON \\ THE OBSERVATIONAL DETERMINATION \\ OF GALACTIC STRUCTURE
}

\author{
C. C. LIN \\ Massachusetts Institute of Technology, U.S.A. \\ C. YUAN \\ City College of New York, U.S.A. \\ and \\ W. W. ROBERTS \\ University of Virginia, U.S.A.
}

(Presented by C. Yuan)

\section{The Plan of Investigation}

To investigate the nature and the extent of the influence of a spiral gravitational field on the observational determination of galactic structure, we examine several models of the solar vicinity, including a spiral gravitational field and the accompanying shocks. (These models are described in the Appendix.) We calculate the radial motions and the proper motions that would be observed from the solar vicinity. We then treat these theoretically calculated data by the conventional methods used for analyzing observational data. In this way, we hope to recover the galactic parameters originally adopted in our models. The accuracy of this process is then a measure of the uncertainty in the existing determinations of galactic constants, because of the influence of the spiral gravitational field.

\section{Observational Justification of this Approach}

Before this procedure can be justified, we must examine whether the field of flow calculated is qualitatively of the same nature as the field of stellar motions observed. As it turned out, the outstanding features of the two fields of motion appear to be in general agreement for the model already adopted (Lin et al., 1969), on the basis of a number of other observational data. In this model, the Sun is placed approximately midway between two major spiral arms. The comparison of these fields will be presented below in this section.

In contrast, if the Sun were placed near a major spiral arm, there would have been rather peculiar features noticeable in the field of motion (see Section 4 below), in strong disagreement with observations.

Figure 1 shows the theoretical line-of-sight velocity $v_{l s}$, divided by radial distance $r$, plotted against galactic longitude for three radial distances, $r=1,2,3 \mathrm{kpc}$ respectively. 


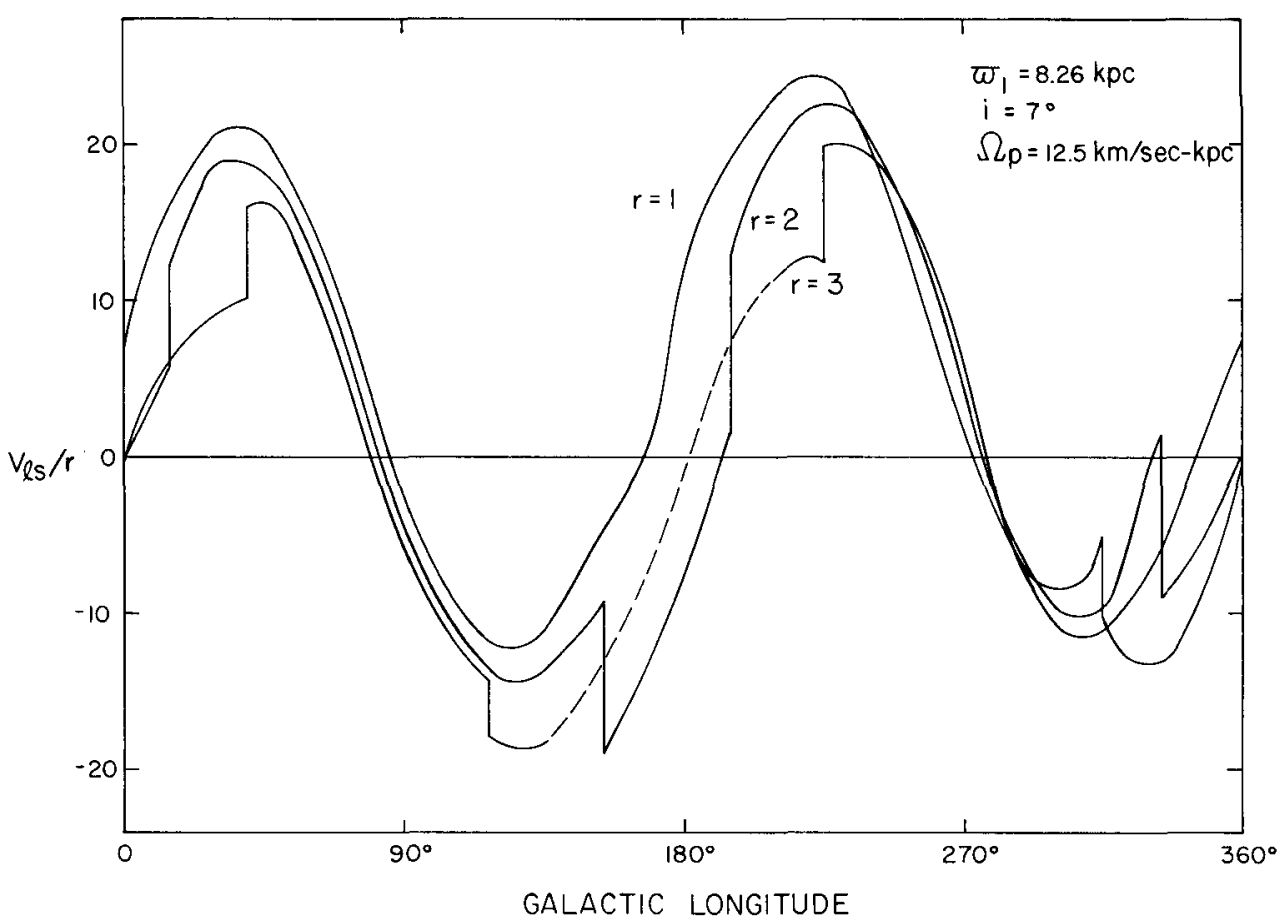

Fig. 1. The plot of $v_{l_{s}} / r$ against galactic longitude, where $v_{l s}$ is the light-of-sight velocity, and $r$ is the radial distance of the star (theory).

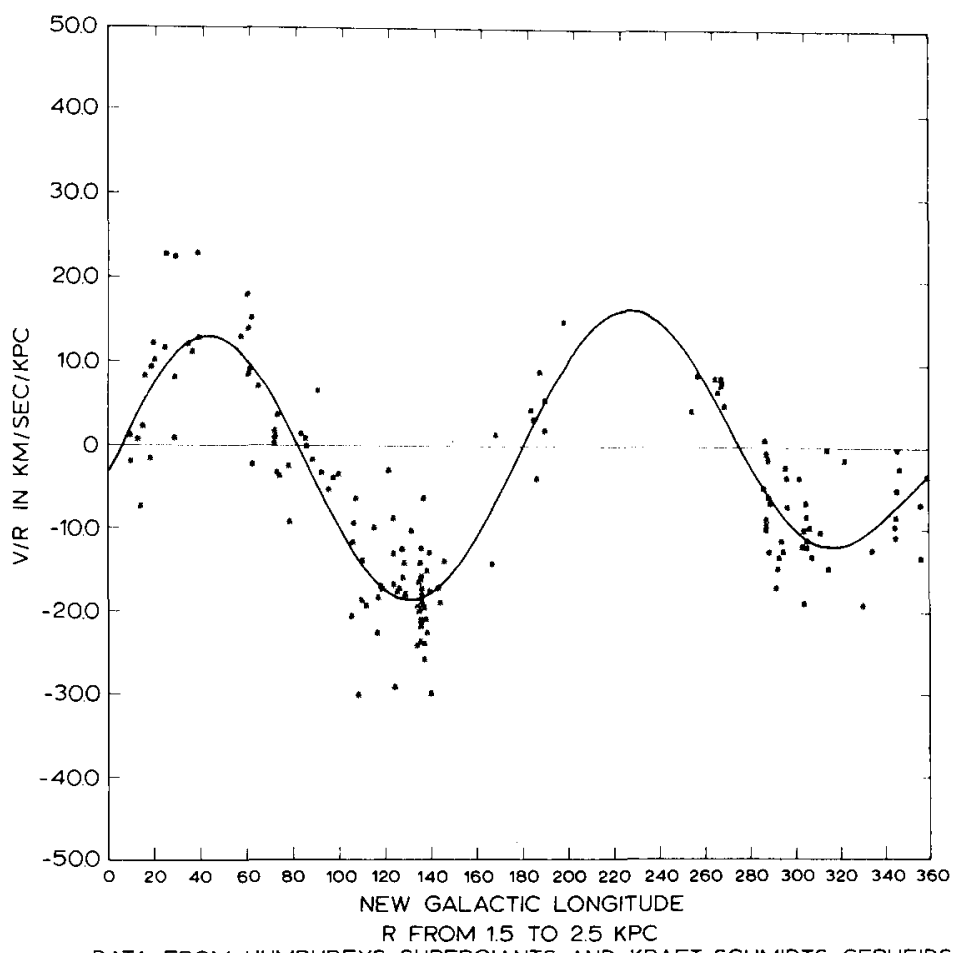

Fig. 2. Same as Figure 1, observation. 

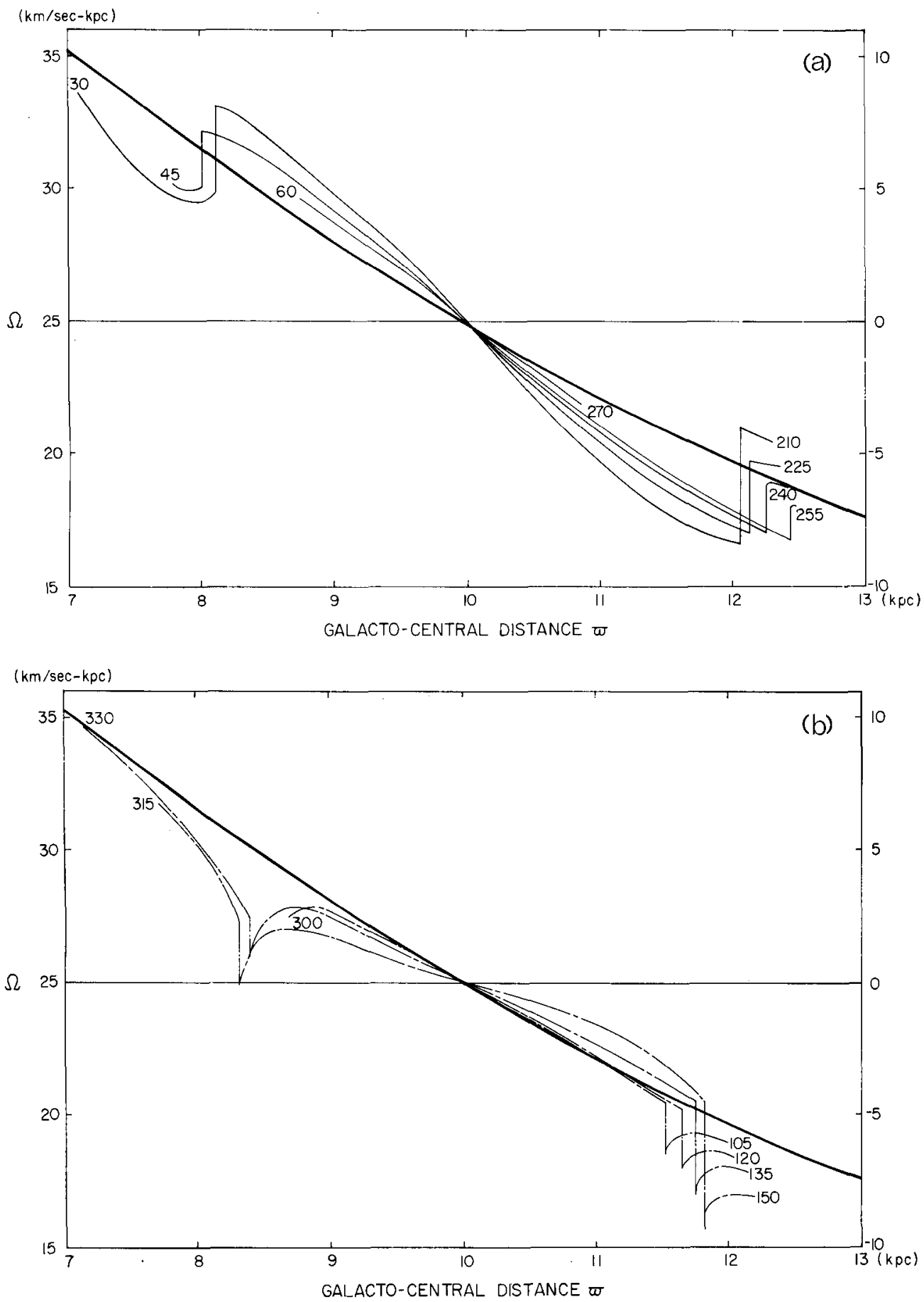

Fig. 3. The rotation curve of the Galaxy and the theoretically expected deviations. (a) First and third quadrants $\left(l=0^{\circ}-90^{\circ}, 180^{\circ}-270^{\circ}\right)$. (b) Second and fourth quadrants $\left(l=90^{\circ}-180^{\circ}, 270^{\circ}-360^{\circ}\right)$. 


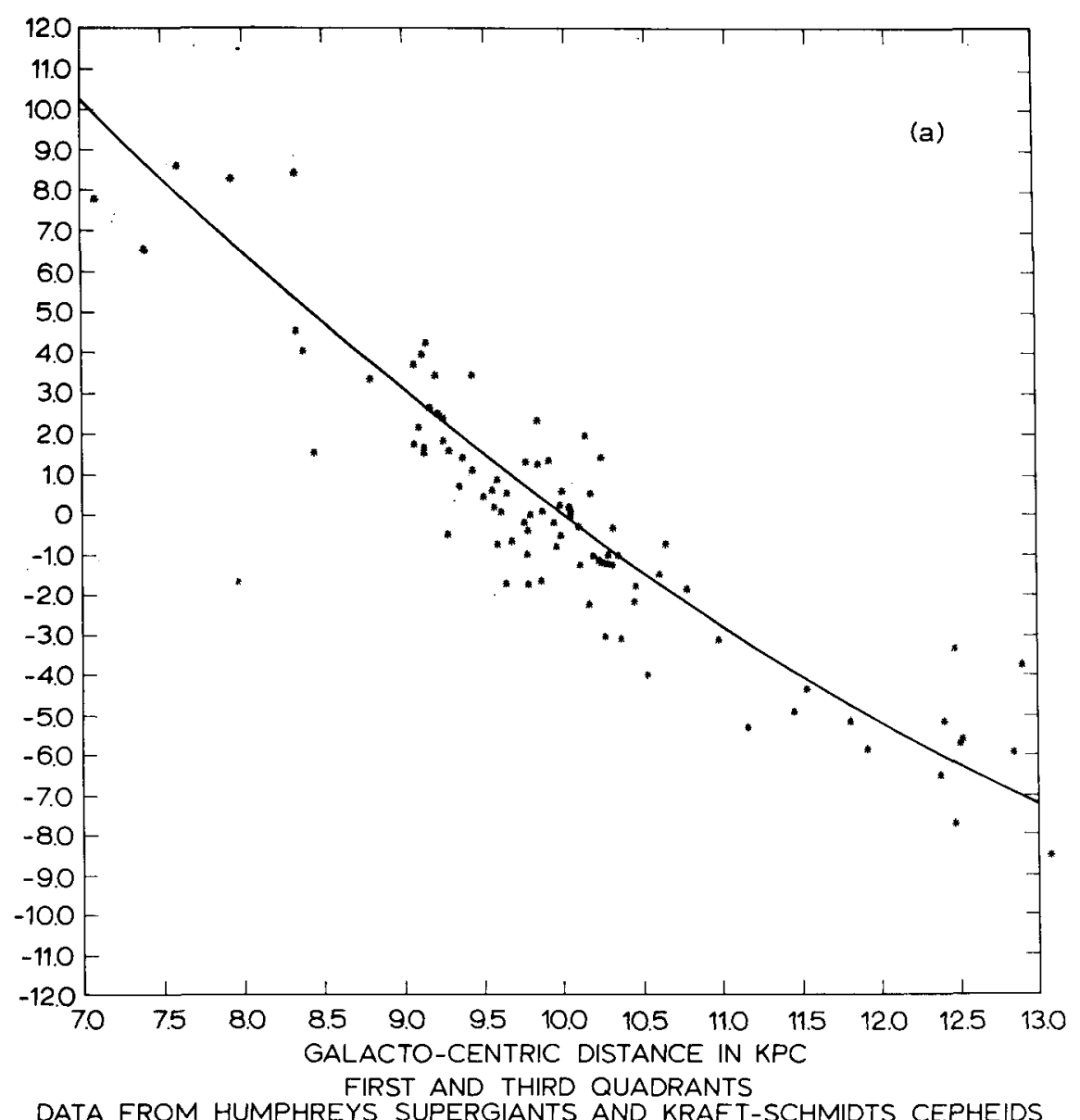

Fig. 4a.

We also prepared a similar plot (Figure 2) of the combined data for early-type stars, cepheids, and supergiants (cf. papers by Kraft and Schmidt, 1963; Rubin et al., 1962, 1964; Humphreys, 1970). Similar characteristics can be noted.

A more clear-cut demonstration of the similarity of the flow fields is shown by Figure 3, where $v_{l s} / \varpi_{0} \sin l$ is plotted against $\pi-\varpi_{0}(\pi=$ galacto-centric distance, whose value $\varpi_{0}$ for the Sun is assumed to be $10 \mathrm{kpc}$ ). The slightly curved thick line is the Schmidt curve. The other curves indicate where the stellar data may be located. From the figure, one can clearly expect the data to drop below the Schmidt curve near $\varpi=12$ and then rise above it at a somewhat larger distance. This is a prominent characteristic noted by the observers, and attributed by them to the peculiar behavior in the Perseus arm. (See Figures $4 \mathrm{a}$ and $4 \mathrm{~b}$, which are reproduced from papers by Kraft and Schmidt, and by Humphreys.) 


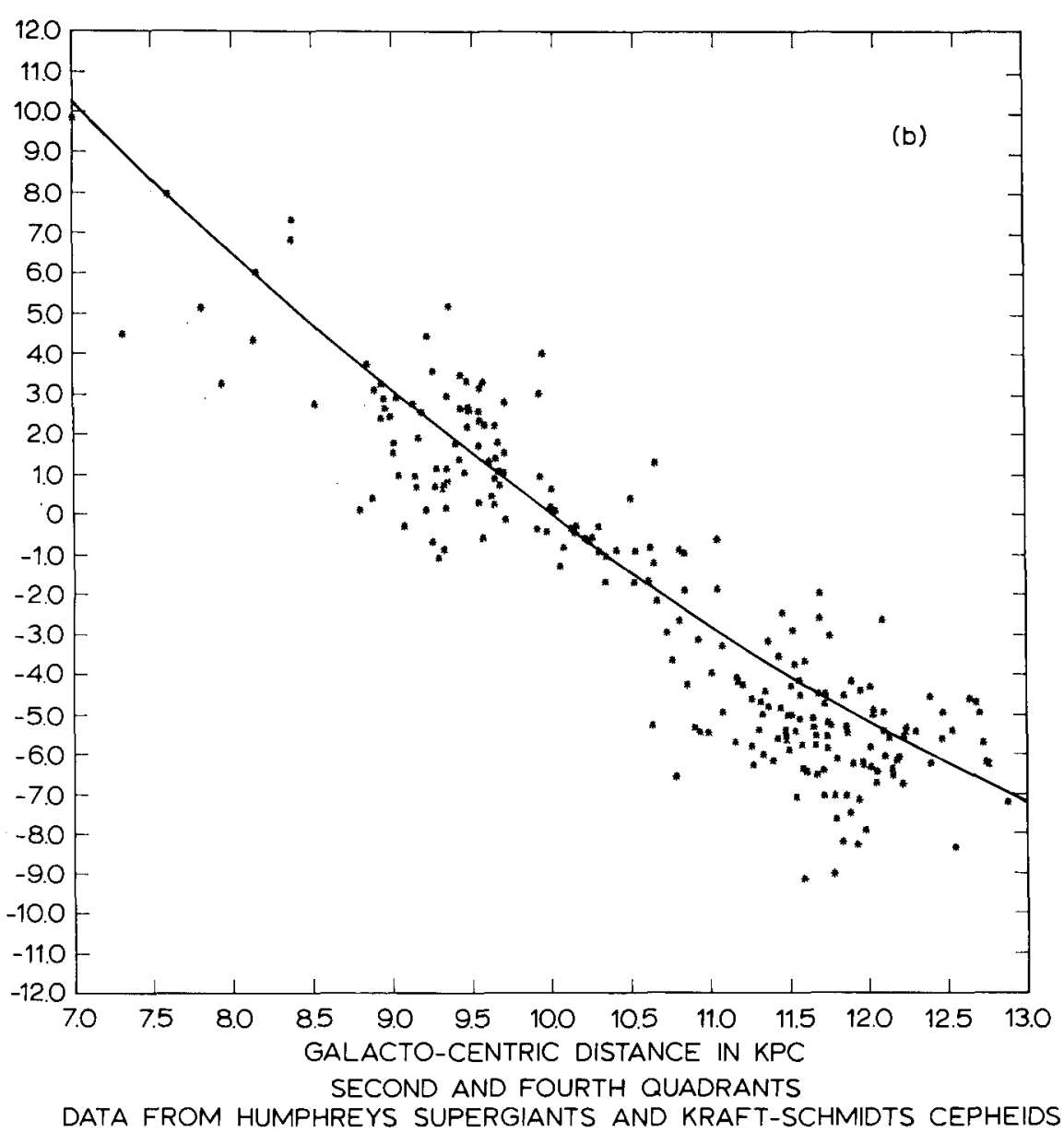

Fig. 4b.

Figs. 4a-b. The rotation curve of the Galaxy and the observed deviations. (a) First and third quadrants. (b) Second and fourth quadrants.

\section{Results}

A summary of the principal results is presented in the following tables. We note that the Oort constants are recovered with good accuracy in the models S-1 and E-1. (These models are described in the appendix).

Especially the determination of

$$
\frac{\Theta}{\bar{\sigma}}=A-B
$$

is blessed with extremely good accuracy. There is a good theoretical reason for this fact. Thus, the influence of the effects we have studied here appears to be below that of the inaccuracy in the observational determination of proper motions. 
We have not yet calculated the curvature term from the theoretical data shown in part in Figure 3. But it is clear from the plot that we would obtain values similar to those obtained by earlier authors. The data show clear upper limits to the curvature term in the rotation curve, but not a clear lower limit.

We have also adopted a method of analysis somewhat more general than the customary ones. We make Fourier analyses of the radial and proper motions at several radial distances from the Sun.

We write

$$
\frac{v_{l s}}{r}=\alpha_{0}+\sum_{n=1}^{\infty}\left(\alpha_{n} \cos n l+\beta_{n} \sin n l\right) .
$$

Two samples of such Fourier coefficients are shown. Clearly, the values of $\beta_{2}$ give good approximations to the Oort constant $A$. The coefficient $\alpha_{0}$ is $K$, and is indeed

\section{TABLE I}

Tabulation of numerical values of $A, B, A-B$,

$$
\boldsymbol{A}+\boldsymbol{B}
$$

1. Oort constant $A$; assumed value $=15 \mathrm{~km} \mathrm{~s}^{-1}$ $\mathrm{kpc}^{-1}$

\begin{tabular}{llll}
\hline & $r=1$ & $r=2$ & $r=3$ \\
\hline S & 14.7 & 14.3 & 13.9 \\
E-1 & 15.2 & 15.1 & 14.7 \\
E-2 & 17.2 & 17.0 & 14.8 \\
S-1 & 16.6 & 16.3 & 15.1 \\
\hline
\end{tabular}

2. Oort constant $B$; assumed value $=-10 \mathrm{~km} \mathrm{~s}^{-1}$ $\mathrm{kpc}^{-1}$

\begin{tabular}{lccc}
\hline & $r=1$ & $r=2$ & $r=3$ \\
\hline S & -10.3 & -10.6 & -10.9 \\
E-1 & -8.8 & -9.5 & -11.1 \\
E-2 & -7.0 & -9.3 & -11.7 \\
S-1 & -7.9 & -9.9 & -11.1 \\
\hline
\end{tabular}

3. Angular velocity $A-B$; assumed value $=$ $=25 \mathrm{~km} \mathrm{~s}^{-1} \mathrm{kpc}^{-1}$

\begin{tabular}{llll}
\hline & $r=1$ & $r=2$ & $r=3$ \\
\hline S & 25.0 & 24.9 & 24.8 \\
E-1 & 24.0 & 24.6 & 25.8 \\
E-2 & 24.2 & 26.3 & 24.5 \\
S-1 & 24.5 & 26.2 & 26.2 \\
\hline
\end{tabular}

4. Radial gradient of circular velocity $A+B$; assumed value $=5 \mathrm{~km} \mathrm{~s}^{-1} \mathrm{kpc}^{-1}$

\begin{tabular}{lrll}
\hline & $r=1$ & $r=2$ & $r=3$ \\
\hline S & 4.4 & 3.7 & 3.0 \\
E-1 & 6.4 & 5.6 & 3.6 \\
E-2 & 10.2 & 7.7 & 3.1 \\
S-1 & 8.7 & 6.4 & 4.0 \\
\hline
\end{tabular}


TABLE IIA

Fourier coefficients for theoretical values of $v_{l s} / r(\mathrm{E}-1)$

\begin{tabular}{lrrr}
\hline $\begin{array}{l}\text { Distance } \\
(\mathrm{kpc})\end{array}$ & \multicolumn{1}{l}{1} & \multicolumn{1}{l}{2} & \multicolumn{1}{l}{3} \\
\hline$\alpha_{0}$ & 4.42 & 4.03 & -0.12 \\
$\alpha_{1}$ & -2.79 & -0.34 & 0.36 \\
$\beta_{1}$ & -1.05 & -2.37 & -3.66 \\
$\alpha_{2}$ & 4.10 & 4.06 & -1.81 \\
$\beta_{2}$ & 15.24 & 15.08 & 14.69 \\
$\alpha_{3}$ & -0.60 & 1.17 & 0.27 \\
$\beta_{3}$ & 0.48 & 0.39 & 0.90 \\
\hline
\end{tabular}

TABLE IIB

Fourier coefficients for theoretical values of $v_{r} / r(\mathrm{~S}-1)$

\begin{tabular}{|c|c|c|c|}
\hline $\begin{array}{l}\text { Distance } \\
(\mathrm{kpc})\end{array}$ & 1 & 2 & 3 \\
\hline$\alpha_{0}$ & 5.00 & 1.81 & -0.16 \\
\hline $\begin{array}{l}\alpha_{1} \\
\beta_{1}\end{array}$ & $\begin{array}{l}-0.96 \\
-1.53\end{array}$ & $\begin{array}{r}1.88 \\
-3.33\end{array}$ & $\begin{array}{r}0.31 \\
-4.10\end{array}$ \\
\hline $\begin{array}{l}\alpha_{2} \\
\beta_{2}\end{array}$ & $\begin{array}{r}4.26 \\
16.57\end{array}$ & $\begin{array}{r}-0.78 \\
16.13\end{array}$ & $\begin{array}{r}-1.13 \\
15.05\end{array}$ \\
\hline $\begin{array}{l}\alpha_{3} \\
\beta_{3}\end{array}$ & $\begin{array}{l}0.17 \\
0.01\end{array}$ & $\begin{array}{r}1.46 \\
-0.50\end{array}$ & $\begin{array}{r}-0.38 \\
0.88\end{array}$ \\
\hline
\end{tabular}

found to agree with the value of $K$ calculated by following density changes along a stream line. The value of $K$ is positive, consistent with our theoretical picture of an expanding motion between spiral arms (cf. Figure 5). Reasons can be given why a smaller value (or even a negative value) is obtained by earlier authors from observational data. Briefly, it is associated with a bias of observational data because the young objects are found predominantly in the spiral arms. This effect can be detected, when Figure 3 is compared in detail with similar plots based on observational data. Fortunately, when the same method of Fourier analysis is applied to observational data, the value of the Oort constant $A$ obtained still agrees approximately with those obtained from the theoretical curves.

\section{Discussion}

To summarize, we have made the following two points:

(1) The systematic deviation of the observed stellar motions from the mean rotation curve shows features similar to those of the streaming motions due to the presence of a spiral gravitational field, provided that we adopt a model similar to that used by Lin et al. (1969). 


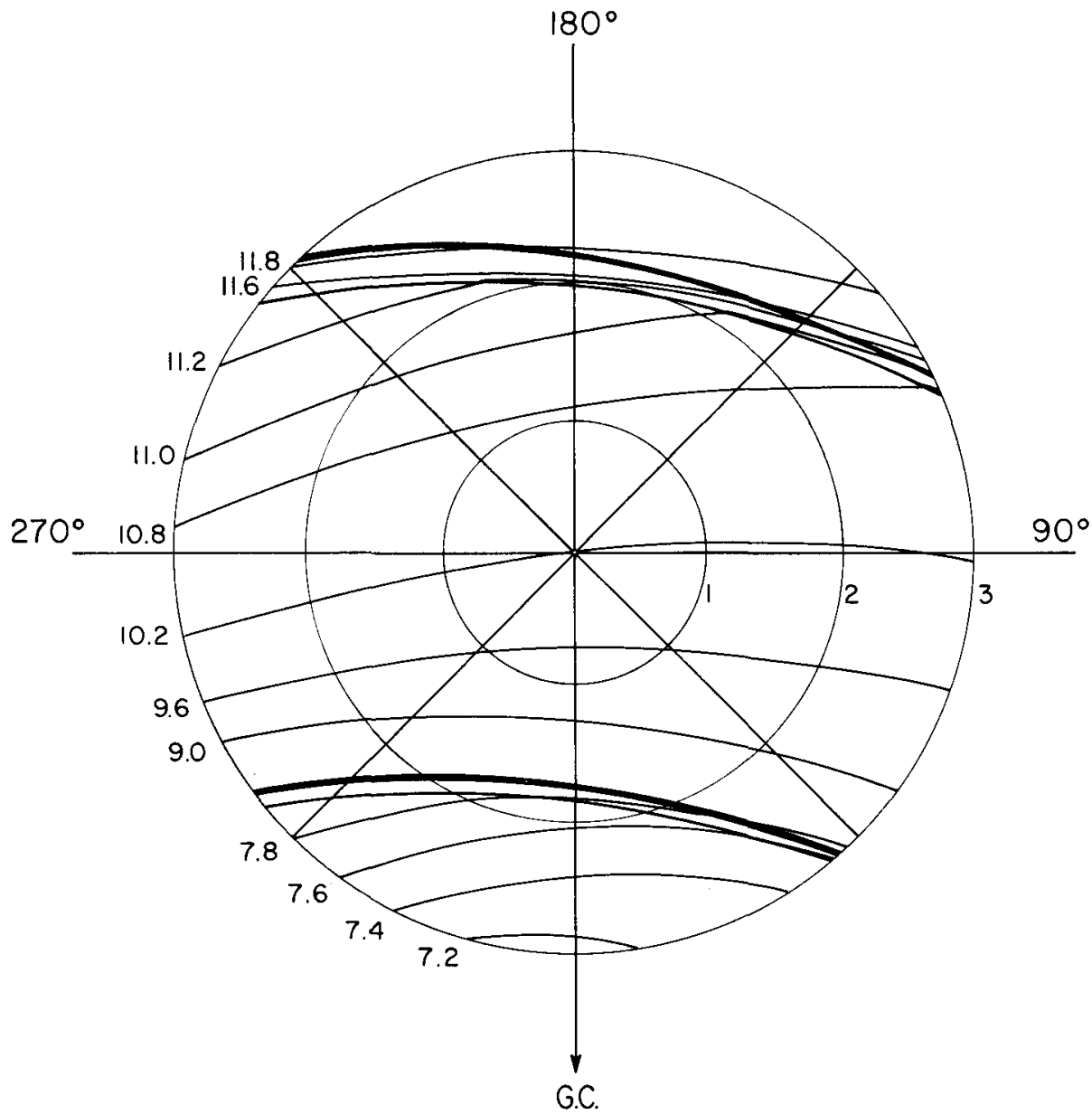

Fig. 5. The flow field in the solar vicinity. Divergence of streamlines indicates that there is expansion in the solar vicinity.

(2) The conventional methods of analysis of stellar motions yield a quite accurate model of the rotation curve of the Galaxy. The determination of Oort constants is not much influenced by the spiral gravitational field.

To gain a better overall perspective, we must ask ourselves what consequences would follow if a different model were adopted. We know that the Sun is located at the inner side of the Orion arm. However, we have insisted that the Orion arm is not a major spiral arm because there is a paucity of important $\mathbf{H}$ II regions. Similarly for the Carina arm. Let us see what the consequences are if the Sun were close to a major arm. If the center of a major arm were placed at $10.3 \mathrm{kpc}$, the diagrams in Figures 1 and 3 would look like Figures 6 and 7. Especially in Figure 6, we note that the doublesine nature of the curve is completely lost, in sharp contrast with well-known obser- 


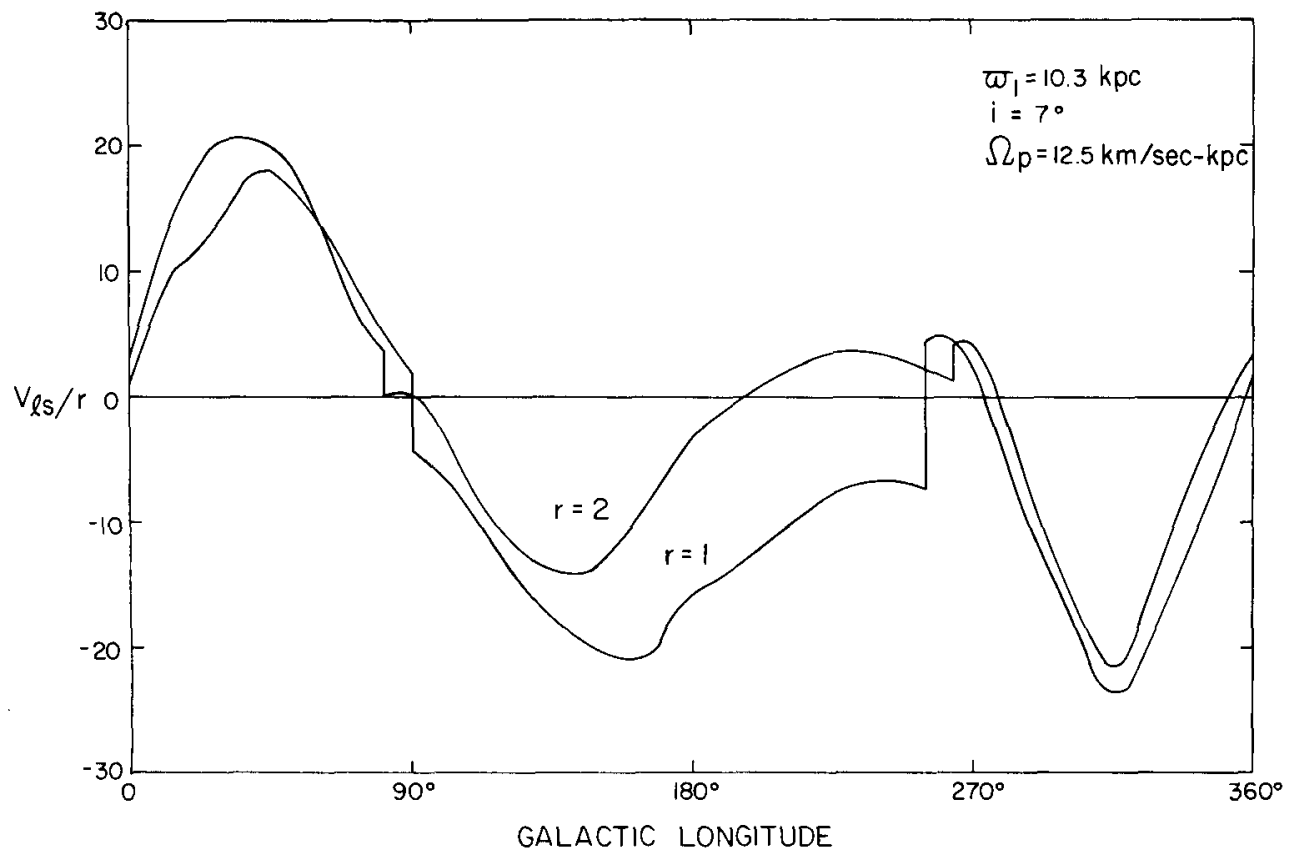

Fig. 6. Same plot as Figure 1 for a model with the Sun close to a major spiral arm. The double-sine character of the curve is suppressed.

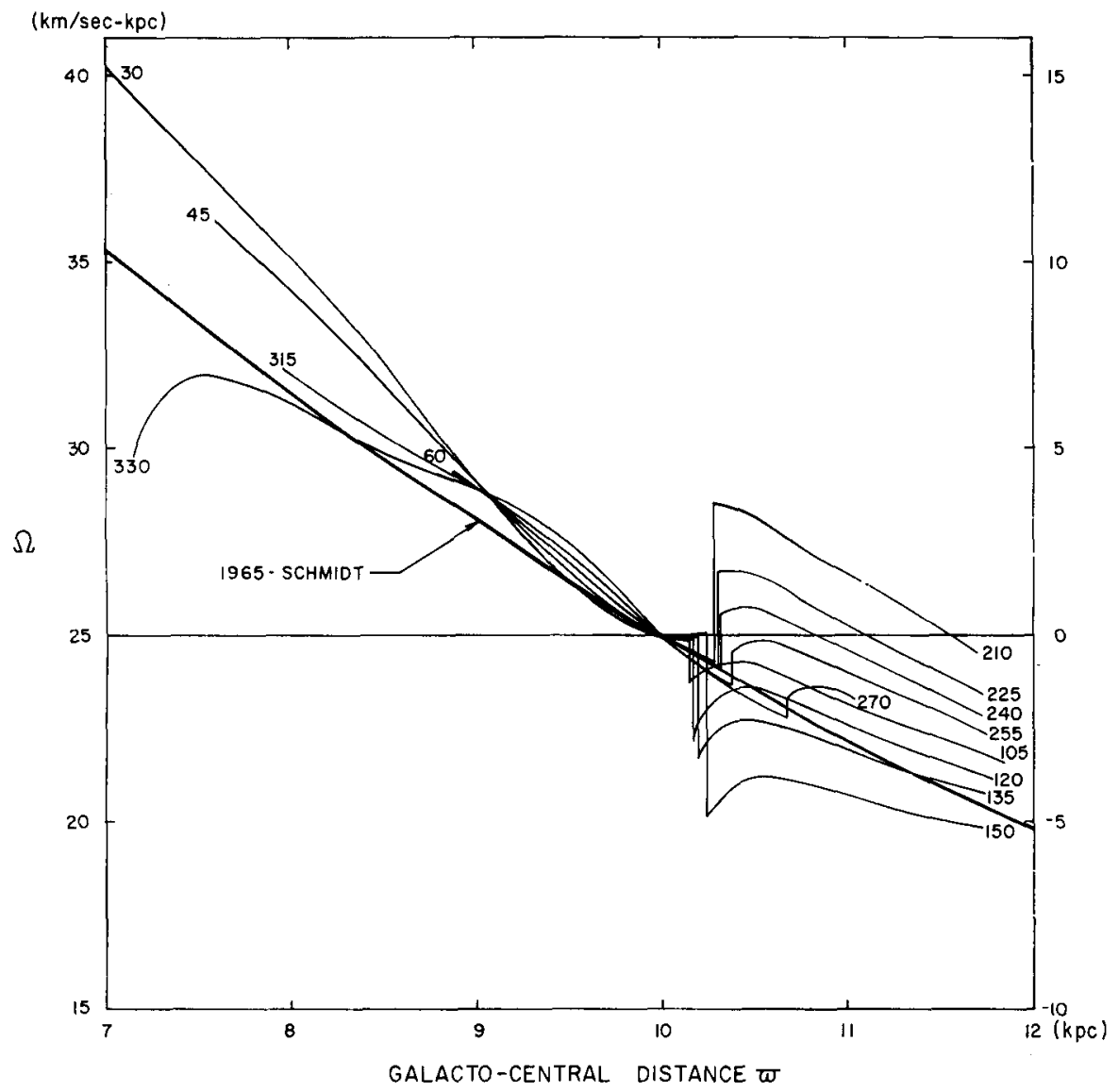

Fig. 7. Same plot as Figure 3 for the model of Figure 6. Note the difference with Figure 3. https://doi.org/10.1017/S1539299600002161 Published online by Cambridge University Press 
vational data. Thus, the good agreement between the theoretical and observed streaming motions obtained in the present study also lends general support to the model adopted in earlier studies.

\section{Appendix - The Models}

Five theoretical models were investigated. These are designated S-1, S-2, S-3 and E-1, E-2. The spirals in the $S$ series have a pitch angle of seven degrees; the $E$ series, eight degrees. The location of the Sagittarius and the Perseus arms are given in the following table

\begin{tabular}{lll} 
& $\begin{array}{l}\text { Sagittarius arm } \\
\text { (in kpc, from G.C.) }\end{array}$ & $\begin{array}{l}\text { Perseus arm } \\
\text { (in kpc, from G.C.) }\end{array}$ \\
\hline S-1 & 8.26 & 12.3 \\
S-2 & 8.5 & 12.55 \\
S-3 & 7.0 & 10.3 \\
& & \\
E-1 & 7.9 & 12.4 \\
E-2 & 7.4 & 11.5 \\
\hline
\end{tabular}

\section{Acknowledgement}

This work is supported in part by the National Science Foundation.

\section{References}

Humphreys, Roberta M.: 1970, Astron. J. 75, 602.

Kraft, R. P. and Schmidt, M.: 1963, Astrophys. J. 137, 249.

Lin, C. C., Yuan, C., and Shu, F. H.: 1969, Astrophys. J. 155, 721.

Rubin, V. C., Burley, J., Kiasatpoor, A., Klock, B., Pease, G., Rutscheidt, E., and Smith, C.: 1962, Astron. J. 67, 491.

Rubin, V. C. and Burley, J.: 1964, Astron. J. 69, 80.

\section{DISCUSSION}

Buscombe: (1) A new finding list of 1569 supergiants is available from me at the Northwestern University. Colour excesses and radial velocities are still needed for nearly half of them. (2) Formally, I have shown (Observatory, 1971) that the kinematics of young luminous galactic clusters is fitted by algebraically larger $A$ and $B$ than the older, less luminous open clusters.

Yuan: Of course, one has to look at the actual distribution of these young galactic clusters in their longitudes and distances before making any fair remark. The selection effect is not very strong here. From our theoretical study, young stars clustered near the shock region indeed tend to give a relatively higher value of $A$. This certainly is consistent with your findings. 